\title{
NEW IRIS RETRACTOR FOR USE IN CRYOSURGERY*†
}

\author{
BY \\ G. M. MACLURE \\ Birmingham and Midland Eye Hospital
}

DURING cryoextraction of the cataractous lens, difficulty may be experienced in manipulating the iris, when applying the iris hook or retractor. Rubenstein (1967) observed that the lens capsule may be broken as the retractor is moved across the lens to expose the upper pole. There may also be difficulty in engaging the iris in the hook if the curved surfaces are too close together. This may, in addition, give rise to difficulty in disengaging the hook after extraction of the lens. Further difficulty may arise because the hook has to be moved across the eye (and hence the anterior surface of the vitreous) to allow the iris to take up its normal position before it can be disengaged. Rubinstein stated that the retractor might be inadvertently depressed and damage the vitreous face above, and Krwawicz (1963) thought that the vitreous loss in two out of five of his cases was probably due to an injury inflicted on the anterior surface of the vitreous with an iris retractor. Either side of the updrawn iris may become frozen to the cryotip, but Rubinstein is of the opinion that this can be avoided if the "head" of the retractor is broad enough to make the sides of the pupil nearly parallel.

To overcome all these difficulties, a new iris retractor $\ddagger$ has been designed (Figure).

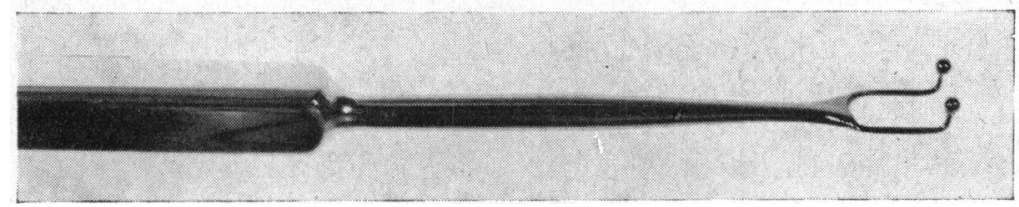

FIGURE. - The iris retractor. $\times 2$.

Extensive trials have been carried out at the Birmingham and Midland Eye Hospital, and the Selly Oak and Queen Elizabeth Hospitals, Birmingham.

\section{Technique}

The two arms of the retractor are placed within the pupil and just touching the lens, the smooth ball-ends preventing damage to the lens capsule and also acting as a slight hook. If the pupil is too small to admit both arms at the same time, one can be engaged at either the 3 or 9 o'clock position and then moved laterally to allow the other arm to engage the opposite pupil margin.

The retractor is then gently moved across the surface of the lens to the $12 \mathrm{o}$ 'clock position as far as the limbal incision, thus exposing the upper pole of the lens.

As the lens is lifted after application of the cryotip and delivery proceeds, or after delivery of the lens, the retractor is easily and quickly disengaged by simply lifting it gently from the eye, allowing the iris to slip back into position, thus protecting the vitreous face. No

* Received for publication January 2, 1967.

$\ddagger$ Now made by Dixey and Sons Ltd., London. 
movement of the retractor across the eye (and hence across the anterior surface of the vitreous) is necessary to disengage the retractor.

The advantages of the retractor are:

(1) Engagement and disengagement of the iris are easy and can be performed by a relatively inexperienced assistant, or by the surgeon himself if preferred.

(2) There is no risk of damaging the lens capsule.

(3) The distal ends of the two arms are wide enough to give good exposure of the upper pole of the lens and to keep the iris away from the cryotip.

(4) The vitreous face is protected as the lens delivery proceeds because the iris retractor can be easily withdrawn without an "unhooking" movement allowing the iris to fall back into position.

(5) A dilated pupil is not essential, as first one arm and then the other can be hooked under the iris sphincter.

(6) The field of vision is not obscured by the instrument and any pressure on the sclera, seen as scleral indentation by one or both arms, is clearly visible.

\section{Summary}

A new iris retractor, primarily for use in cryosurgery, is described. The operative technique and some advantages are given.

I wish to express my gratitude to Mr. L. P. Jameson Evans for the initial stimulus to design this retractor. I should also like to thank Mr. M. J. Roper-Hall, Mr. K. Rubinstein, Mr. B. Kesby, and Mr. I. A. R. Brown, who have used the instrument, for their comments, Mr. Peter Elder (Dundee) for making the original prototype, and Mr. G. D. Holland for the photograph.

\section{REFERENCES}

KRWAWICZ, T. (1963). Brit. J. Ophthal., 47, 36.

RUBINSTEIN, K. (1967). I Ibid., 51, 178. 